\title{
Does perioperative oral rofecoxib therapy improve functional recovery after knee replacement surgery?
}

Buvanendran A, Kroin JS, Tuman KJ, Lubenow TR, Elmofty D, Moric $M$, et al. Effects of perioperative administration of a selective cyclooxygenase 2 inhibitor on pain management and recovery of function after knee replacement: a randomized controlled trial. JAMA 2003;290:2411-8.

Background: Managing pain after total knee replacement is important for clinical outcome. While NSAIDs can decrease the inflammatory response associated with surgery, their use has been limited by concerns of bleeding in the early postoperative period. Selective cyclooxygenase-2 (COX-2) inhibitors have little or no effect on coagulation and have been approved for the treatment of acute postoperative pain. However, their efficacy on outcomes other than pain has not been well reported.

Design: This randomized controlled blinded trial involved consecutive patients undergoing elective total knee replacement. Patients were randomly assigned to receive rofecoxib orally $(50 \mathrm{mg}$ 1 day and 1-2 hours preoperatively and daily for 5 days after surgery, and $25 \mathrm{mg}$ on postoperative day 6 for 8 days) or placebo. The primary outcome was pain control; also measured were occurrences of nausea and vomitang, postoperative knee range of motion (active and passive), sleep disturbances and patient satisfaction. The study was designed to detect a $20 \%$ difference in opioid consumption (study power $>90 \%, \alpha=0.05)$. An intentionto-treat analysis was performed
Results: Of the 254 patients assessed, 145 declined participation and 39 met exclusion criteria, which left 70 patients who were ultimately included (35 in the rofecoxib group and 35 in the placebo group). Sixty-six patients (94\%) completed the study. Patients had similar surgical durations, intraoperative parameters and fluid therapies. The results are summarized in Table 1. There were no bleeding complications in either group.

Commentary: The use of COX-2 inhibitors following orthopedic surgery has been the topic of much debate. Experimental data suggest that these drugs prevent bone formation and fracture healing, ${ }^{1}$ which limits their use in fracture surgery. The use of rofecoxib has also revealed limited benefit in pain control following prostate surgery; ${ }^{2}$ however, recent randomized trials have reported significant reductions in pain after hip and knee arthroplasty with the selective COX-2 inhibitors parecoxib and valdecoxib. ${ }^{3,4}$ The strengths of the rofecoxib study include the randomization of consecutive patients and the use of double-blinding techniques. Although the reduction in opioid requirements with rofecoxib is compelling, the mean $8.5^{\circ}$ in-

Table 1: Outcomes following perioperative administration of oral rofecoxib therapy or placebo in patients undergoing total knee replacement

\begin{tabular}{lccc}
\hline Outcome & $\begin{array}{c}\text { Placebo group } \\
n=35\end{array}$ & $\begin{array}{c}\text { Rofecoxib group } \\
n=35\end{array}$ & $p$ value \\
\hline Volume of postoperative epidural anesthetic required, mL (SD) & $302.6(62.7)$ & $252.2(65.5)$ & 0.003 \\
Degree of pain 1 wk after surgery, VAS score (SD) & $3.7(2.9-4.7)$ & $2.6(1.4-3.5)$ & 0.03 \\
Incidence of vomiting, no. (\%) & $9(26)$ & $2(6)$ & 0.047 \\
Sleep disturbance* on third night after surgery, median & $4(2-5)$ & $0(0-0)$ & $<0.001$ \\
score (SD) & $100.8(11.8)$ & $109.3(8.5)$ & 0.01 \\
Degree of knee flexion 1 mo after surgery, mean (SD) & & &
\end{tabular}

Note: $\mathrm{SD}=$ standard deviation, $\mathrm{VAS}=$ visual analogue scale

*Sleep disturbance was rated on a 10 -point scale: $0=$ no sleep disturbance, $10=$ greatest sleep disturbance.

crease in range of motion 1 month after surgery is not. The investigators do not report any data that support their reliability in assessing knee range of motion during follow-up. We do not know whether range of motion was measured by the same people or by trained physical therapists. Measurement error could inflate (or decrease) the observed benefit of rofecoxib.

Practice implications: On the basis of the results from this trial, clinicians can inform their patients undergoing total knee replacement that oral rofecoxib therapy reduces analgesic requirements, improves sleep and reduces episodes of postoperative vomiting. However, additional data on the reliability of measuring range of motion are needed before extending its efficacy to patient function. In addition, clinicians should not extrapolate this study's short-term results to longer term outcomes following COX-2 inhibitor use.

Mohit Bhandari

Department of Clinical

Epidemiology and Biostatistics

Division of Orthopaedic Surgery

McMaster University

Hamilton, Ont.

\section{References}

1. Simon AM, Manigrasso MB, O'Connor JP. Cyclo-oxygenase 2 function is essential for bone fracture healing. 7 Bone Miner Res 2002;17:963-76.

2. Huang JJ, Taguchi A, Hsu H, Andriole GL Jr, Kurz A. Preoperative oral rofecoxib does not decrease postoperative pain or morphine consumption in patients after radical prostatectomy: a prospective, randomized, double-blinded, placebo-controlled trial. 7 Clin Anesth 2001;13:94-7.

3. Malan TP Jr, Marsh G, Hakki SI, Grossman E, Traylor L, Hubbard RC. Parecoxib sodium, a parenteral cyclooxygenase 2 selective inhibitor, improves morphine analgesia and is opioid-sparing following total hip arthroplasty. Anesthesiology 2003;98:950-6.

4. Reynolds LW, Hoo RK, Brill RJ North J, Recker DP, Verburg KM. The COX-2 specific inhibitor, valdecoxib, is an effective, opioid-sparing analgesic in patients undergoing total knee arthroplasty. 7 Pain Symptom Manage 2003;25:133-41. 